\title{
Discovery of an eccentric 30 day period in the supergiant X-ray binary SAX J1818.6-1703 with INTEGRAL *
}

\author{
J. A. Zurita Heras and S. Chaty \\ Laboratoire AIM, CEA/DSM-CNRS-Université Paris Diderot, IRFU/Service d'Astrophysique, 91191 Gif-sur-Yvette, France \\ e-mail: [juan-antonio.zurita-heras; sylvain.chaty]@cea.fr
}

Received 17 October 2008 / Accepted 15 November 2008

ABSTRACT

\begin{abstract}
Context. SAX J1818.6-1703 is a flaring transient X-ray source serendipitously discovered by BeppoSAX in 1998 during an observation of the Galactic centre. The source was identified as a high-mass X-ray binary with an OB supergiant companion (SGXB). Displaying short and bright flares and an unusually very low quiescent level implying an intensity dynamical range as large as $10^{3-4}$, the source was classified as a supergiant fast X-ray transient (SFXT).

Aims. The mechanism triggering the different temporal behaviour observed between the classical SGXBs and the recently discovered class of SFXTs is still debated. The discovery of long orbits ( $>15 \mathrm{~d}$ ) should help to discriminate between emission models and bring constraints.

Methods. We analysed archival INTEGRAL data on SAX J1818.6-1703. We built short- and long-term light curves and performed a timing analysis in order to study the temporal behaviour of SAX J1818.6-1703 on different time scales.

Results. INTEGRAL revealed an unusually long orbital period of $30.0 \pm 0.2 \mathrm{~d}$ and an elapsed accretion phase of $\sim 6 \mathrm{~d}$ in the transient SGXB SAX J1818.6-1703. This implies an elliptical orbit and constraints the possible supergiant spectral type between B0.5-1I with eccentricities $e \sim 0.3-0.4$ (for the average fundamental parameters of supergiant stars). During the accretion phase, the source behaved like a classical SGXB. The huge variations of the observed X-ray flux can be explained through accretion of macro-clumps formed within the stellar wind. Our analysis strengthens the model which predicts that SFXTs behave as SGXBs but with different orbital parameters, thus different temporal behaviour.
\end{abstract}

Key words. X-rays: binaries - X-rays: individuals: SAX J1818.6-1703

\section{Introduction}

Supergiant/X-ray binaries (SGXBs) are high-mass X-ray binaries (HMXB) composed of an $\mathrm{OB}$ supergiant companion star and a compact object (a neutron star (NS) or a black hole). In SGXBs, the compact object orbits the massive companion within a few days (3-15 d) in circular (or slightly eccentric) orbits. Supergiant stars possess a strong stellar wind that is partly captured by the compact object, producing X-ray radiation. This happens either by direct spherical accretion or by Roche-Lobe overflow via an accretion disk on the compact object. As the compact object always orbits within the stellar wind, these systems are persistent $\mathrm{X}$-ray emitting objects and show high variations on short time scales.

Very few of these systems were known before INTEGRAL's launch (Lutovinov et al. 2005; Dean et al. 2005; Bodaghee et al. 2007). The mission allowed the discovery of several new sources that could be identified as SGXBs (e.g. Walter et al. 2006; Chaty et al. 2008). These sources show typical hard X-ray spectra of accreting pulsars and most of them show a strong absorption leading to their classification as obscured HMXB (e.g. Filliatre \& Chaty 2004; Rodriguez et al. 2006; Bodaghee et al. 2006; Zurita Heras et al. 2006). Among the new SGXBs, INTEGRAL has revealed a new subclass. Indeed, several newly discovered

\footnotetext{
^ Discovery first reported at the 7th INTEGRAL workshop (Zurita Heras et al., in press). Based on observations with INTEGRAL, an ESA project with instruments and science data centre funded by ESA member states (especially the PI countries: Denmark, France, Germany, Italy, Switzerland, Spain), Czech Republic and Poland, and with the participation of Russia and the USA.
}

sources were identified as Galactic X-ray sources with a supergiant companion and displaying a transient behaviour (e.g. IGR J17544-2619, in't Zand 2005; Pellizza et al. 2006). The quiescent level in these systems is of the order $10^{32-33} \mathrm{erg} \mathrm{s}^{-1}$ (e.g. IGR J08408-4503 Götz et al. 2007; Leyder et al. 2007). The X-ray luminosity increases up to $10^{36} \mathrm{erg} \mathrm{s}^{-1}$ (as observed in known SGXBs) only during periods of short and luminous flares. The X-ray luminosity remains at a very low level (if not totally absent) during most of the time, except during the flares. These flaring periods last a few hours at most and then the source goes back to an undetectable level of emission (e.g. XTE J1739-302, Smith et al. 2006). Therefore, they received the name of Supergiant Fast X-ray Transient (SFXT, Negueruela et al. 2006). Besides their transient nature, the spectral features of SFXTs are similar to the previously known persistent SGXBs.

BeppoSAX discovered the new X-ray transient SAX J1818.6-1703 on March 11, 1998, during a bright flare (in 't Zand et al. 1998) ${ }^{1}$. in't Zand (2005) reported its possible association with other fast X-ray transients like XTE J1739-302 and IGR J17544-2619, archetypes of SFXTs. Beside detections during deep surveys of the Galactic centre (Revnivtsev et al. 2004; Kuulkers et al. 2007), similar short (a few hours) and bright flares of SAX J1818.6-1703 were also detected by INTEGRAL (see Table 1, Grebenev \& Sunyaev 2005; Sguera et al. 2005; Grebenev \& Sunyaev 2008). Grebenev \& Sunyaev (2005) showed that the flares had a complex structure with a short 10-20 min precursor peak and a main $1.5-2 \mathrm{~h}$ long peak with different intensities. The hard X-ray spectrum

\footnotetext{
${ }^{1}$ All fluxes reported in the literature are summarised in Table 1.
} 
Table 1. Summary of previous detections of SAX J1818.6-1703.

\begin{tabular}{ccc}
\hline $\begin{array}{c}\text { Energy range } \\
\text { keV }\end{array}$ & $\begin{array}{c}\text { Flux } \\
\text { mCrab }\end{array}$ & Phase \\
\hline $2-9^{1}$ & 100 & $0.24-0.28$ \\
$9-25^{1}$ & 400 & $0.24-0.28$ \\
$18-60^{\star}$ & 4.9 & - \\
$18-45^{2}$ & 230 & $0.10-0.13$ \\
$45-70^{2}$ & 170 & $0.10-0.13$ \\
$20-30^{3}$ & 178 & 0.12 \\
$20-30^{3}$ & 185 & 0.16 \\
$0.5-10^{4}$ & $7.5 \pm 0.1^{\ddagger}$ & $0.92-0.96$ \\
$20-60^{\circ}$ & $11.1 \pm 0.9$ & - \\
$60-150^{\circ}$ & $29.0 \pm 2.5$ & - \\
$18-45^{5}$ & 40 & $0.06-0.08$ \\
$0.5-10^{6}$ & $<0.11^{\ddagger}$ & $0.56-0.59$ \\
$2-10^{7}$ & $4.2^{\ddagger}$ & $0.13-0.17$ \\
\hline
\end{tabular}

${ }^{\ddagger}$ In units of $10^{-12} \mathrm{erg} \mathrm{cm}^{-2} \mathrm{~s}^{-1} ;{ }^{1}$ BeppoSAX, 1998-03-11 (in 't Zand et al. 1998); ${ }^{\star}$ INTEGRAL, Aug.-Sept. 2003 (Revnivtsev et al. 2004); ${ }^{2}$ INTEGRAL, 2003-09-09 (Grebenev \& Sunyaev 2005); ${ }^{3}$ INTEGRAL, 2003-10-09 and 10 (Sguera et al. 2005); ${ }^{4}$ Chandra, 2006-09-19, exp. 10 ks (in't Zand et al. 2006); ${ }^{\circ}$ INTEGRAL, Feb.-Apr.+Aug-Oct. 2005 and Feb.-Apr. 2006 (Kuulkers et al. 2007); ${ }^{5}$ INTEGRAL, 2008-04-15 and 16 (Grebenev \& Sunyaev 2008); ${ }^{6}$ XMM-Newton, 2006-10-08, exp. 13 ks (Bozzo et al. 2008); ${ }^{7}$ Swift, 2008-04-18, exp. 2 ks (Bozzo et al. 2008).

(fitted with simple analytical models such as a power law) was also variable and became harder during the long flares. Using all INTEGRAL public data available before March 2007, Walter \& Zurita Heras (2007) listed 11 flares with duration of 2-6 ks, and showed that SAX J1818.6-1703 was always detected and displayed an intensity dynamic range of 250 between the lowest detection in deep mosaics and the brightest detection in individual pointings. in't Zand et al. (2006) and Bozzo et al. (2008) reported a quiescent low level of $\sim(4-8) \times$ $10^{-12} \mathrm{erg} \mathrm{cm}^{-2} \mathrm{~s}^{-1}$ with Chandra and Swift, and an unabsorbed $0.5-10 \mathrm{keV}$ flux $3 \sigma$ upper limit of $1.1 \times 10^{-13} \mathrm{erg} \mathrm{cm}^{-2} \mathrm{~s}^{-1}$ with XMM-Newton implying an intensity dynamic range of $10^{3-4}$, as large as in other SFXTs. The Chandra spectrum was fitted with an absorbed power law: $N_{\mathrm{H}}=(6.0 \pm 0.7) \times 10^{22} \mathrm{~cm}^{-2}$ and $\Gamma=1.9 \pm 0.3$.

As it was similar to other SFXTs, while searching for a reddened early-type star in optical and infrared catalogues, Negueruela \& Smith (2006) proposed the source 2MASS J18183790-1702479 as the likely counterpart. Confirmed by Chandra, the best X-ray position is RA (2000) = $18^{\mathrm{h}} 18^{\mathrm{m}} 37.9^{\mathrm{s}}$ and Dec. $=-17^{\circ} 02^{\prime} 47.9^{\prime \prime}\left(0.6^{\prime \prime}\right.$ at $90 \%$ confidence level in't Zand et al. 2006). The companion spectral type was constrained to a O9-B1 supergiant star (Negueruela \& Schurch 2007).

In this study, we used the public INTEGRAL data in order to look for all the rare and short flares of SAX J1818.6-1703 and to study the long-term behaviour of the source, particularly its faint activity. In Sect. 2, we present the observations, the data analysis, the creation of deep mosaics and the timing analysis. In Sect. 3, we present the results and discuss the implications in Sect. 4.

\section{Observations and data analysis}

Launched in 2002 on a 3-day eccentric orbit, the ESA space mission INTErnational Gamma-Ray Astrophysics Laboratory (INTEGRAL, Winkler et al. 2003) carries 4 instruments onboard, of which 3 are dedicated to the detection of high-energy photons with good angular and spectroscopic resolutions; the last one is an optical $V$-band telescope. In this work, we used data from the hard X-ray and soft $\gamma$-ray coded-mask imager IBIS/ISGRI whose main characteristics are a $15 \mathrm{keV}-1 \mathrm{MeV}$ energy range, a wide field of view (FOV) of $29^{\circ} \times 29^{\circ}$, and an angular resolution of $12^{\prime}$ (Ubertini et al. 2003; Lebrun et al. 2003).

The observations were divided in individual pointings with an usual exposure time of $\sim 1-5 \mathrm{ks}$. Discarding pointings with exposure times $<600 \mathrm{~s}$ and source off-axis angles $>14^{\circ}$, we analysed 4003 pointings distributed in 191 revolutions between Feb. 2003 (rev. 0046) and Nov. 2007 (rev. 0495). The total exposure time on the source was $9.3 \mathrm{Ms}$ (i.e. $108.4 \mathrm{~d}$ ) that were unequally distributed along the 4-year observations.

The ISGRI data were reduced using the Offline Scientific Analysis version 7.0 software (OSA7). Individual sky images for each pointing were created in the $22-50 \mathrm{keV}$ energy range. The source count rate was extracted in each image (pointings and mosaics) with the OSA tool mosaic_spec (version 1.7) where the position was fixed to the Chandra position and the point-spread function width was fixed to $6^{\prime}$. All detections of the source were considered above the $5 \sigma$ significance level. Once the rare flares were localised, we built deep-exposure sky mosaics where the pointings with detections were discarded. The goal was to observe the faint activity of the source and to constrain the lowest hard X-ray flux. We created mosaics for each revolution with exposures between 78-186 ks. Then, we combined consecutive revolutions in which the source was not detected and when the time gap between two revolutions was shorter than $50 \mathrm{~d}$. That resulted in 14 deep mosaics with exposure between 200-1000 ks (except 2 cases with exposures of 60 and $80 \mathrm{ks}$ ). All the extracted fluxes are gathered to build the light curve of SAX J1818.6-1703 (see Fig. 1). The $22-50 \mathrm{keV}$ count rate can be converted with the relation $1 \mathrm{Crab}=177.5$ counts $\mathrm{s}^{-1}=9.2 \times 10^{-9} \mathrm{erg} \mathrm{cm}^{-2} \mathrm{~s}^{-1}$. The IJD time unit corresponds to MJD = IJD + 51544 .

\section{Results}

The source was detected in 32 individual pointings that were distributed in 10 revolutions (crosses in Fig. 1). The count rate was mostly between $(4.1-19.1) \pm 0.8$ counts $\mathrm{s}^{-1}$. On Sept. 9, 2003, there were 2 particular very bright flares that reached $44.8 \pm$ 0.8 and $39.1 \pm 1.0$ counts $\mathrm{s}^{-1}$ separated by $2 \mathrm{~h}$ (see Grebenev \& Sunyaev 2005). Discarding all these pointings, we looked for the activity of the source around the flares within the same revolution. The source was only detected in 4 out of 10 revolutions with count rates between $(0.9 \pm 0.1)-(1.7 \pm 0.3)$ counts s$^{-1}$ (triangles in Fig. 1, and $5 \sigma$ upper limits when the source was not detected). In the other revolution mosaics, the source was only detected in 2 of them with similar count rates (boxes in Fig. 1). Both detections occurred before or after a revolution with flares. Beside these detections, we looked for other detections, accumulating consecutive revolutions. Even with very long exposure times, the source was not detected with an average $5 \sigma$ upper limit of 0.3 counts $\mathrm{s}^{-1}$ (large $5 \sigma$ upper limits in Fig. 1). In total, the source was detected 8 times for a few days at most. Detections 1-2 and 3-4 were both separated by $\sim 30 \mathrm{~d}$, and 6-7 by $\sim 60 \mathrm{~d}$. The source seems to display a periodic recurrent flaring activity separated by $\sim 30 \mathrm{~d}$ and lasting for $\sim 5 \mathrm{~d}$. However, flares in several observation periods were missed.

To search for the missing flares, we studied the source long-term behaviour using the count rates extracted in pointings and revolution mosaics. In pointings, the sensitivity 


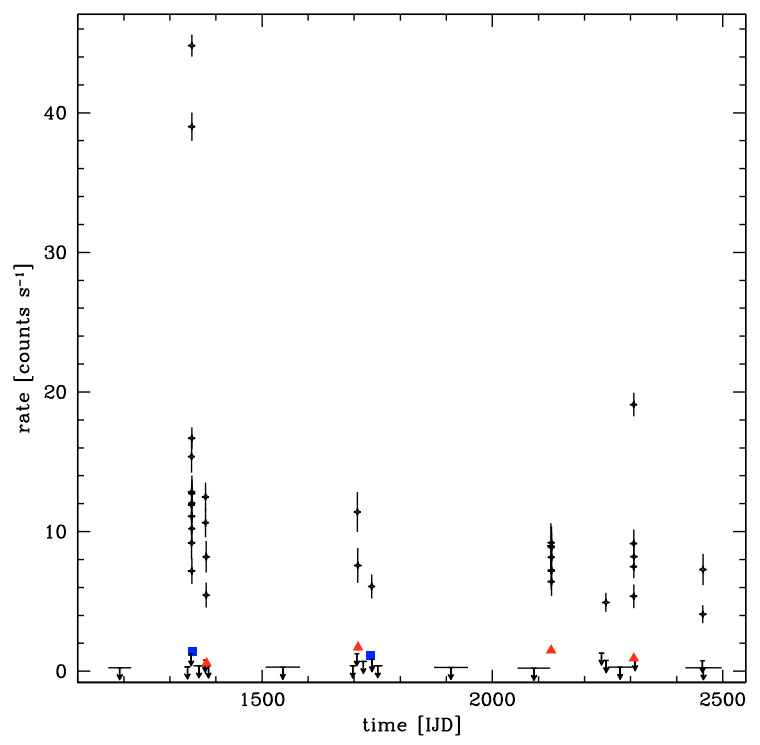

Fig. 1. 22-50 keV light curve of SAX J1818.6-1703. The data points are described in the text.

diminished when the source moved outside of the fully-coded FOV of ISGRI. The $5 \sigma$ level varied between $\sim 6-12$ counts $\mathrm{s}^{-1}$ when the source off-axis angle varied between $9-14^{\circ}$. As the source was located at an off-axis angle $\geq 9^{\circ}$ in $\sim 2 / 3$ of the pointings, the number of flares below 12 counts $\mathrm{s}^{-1}$ was underestimated. In mosaics with exposure $<20 \mathrm{ks}$, the $5 \sigma$ level was usually $>4$ counts $\mathrm{s}^{-1}$, similar to the count rate of the faint detected flares. Therefore, the Lomb-Scargle periodogram (the fast method of Press \& Rybicki 1989) was computed using the data of flares and mosaics with exposure $>20 \mathrm{ks}$. The best period was $30.0 \pm 0.2 \mathrm{~d}$ (uncertainty derived using Eq. (14) of Horne \& Baliunas 1986). We folded the light curve with this period and a time start of IJD 1133.7365 (see Fig. 2). All detections fell between phases $0.0-0.2$, thus, an elapsed time of 6 d. Only 31 out of 191 revolutions fell within the flaring periods. SAX J1818.6-1703 was detected in 12 of them as described above. We built a mosaic with the other 19 revolutions (exp. $744 \mathrm{ks}$ ) and the source was detected with a count rate of $0.47 \pm 0.06$ counts $\mathrm{s}^{-1}$, implying a variation by a factor of 2-4 compared to the average count rates extracted in the 6 revolutions with a detection. Combining revolutions per step of 0.2 phase between $0.2-1.0$ (4 mosaics with exposures of 1.5-2 Ms each), the source was not detected with a $5 \sigma$ upper limit of $\sim 0.2$ counts $\mathrm{s}^{-1}$.

\section{Discussion}

As reported above, SAX J1818.6-1703 was identified as a SGXB with a spectral type between O9I-B1I. Such systems usually radiate with an average luminosity of $10^{36} \mathrm{erg} \mathrm{s}^{-1}$ with an important variability of a factor $\$ 20$ and in which the compact object orbits around the supergiant companion at a distance $\lesssim 2 R_{*}$. However, the source behaved differently compared to other known SGXBs. For SAX J1818.6-1703, we found a period of $30.0 \pm 0.2 \mathrm{~d}$ which can be interpreted as the orbital period of the system. A modulation of activity at a consistent period is found (Smith, private communication) in the RXTE Galactic bulge scan data (Markwardt et al. 2000). As observed by INTEGRAL, the source only radiated for $\sim 6 \mathrm{~d}$ during its orbit. The detections reported by other missions are in good agreement with our ephemeris (see last column in Table 1).

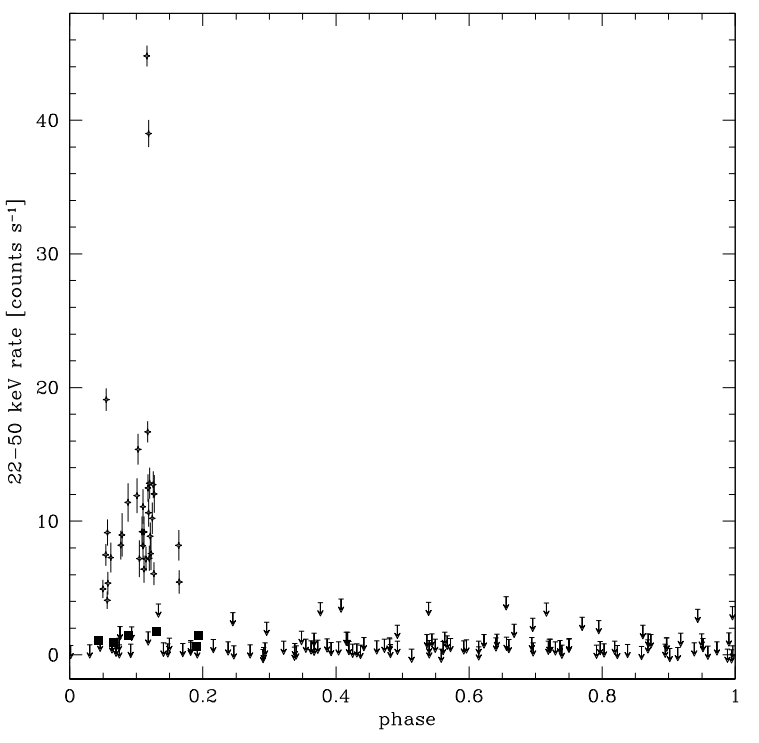

Fig. 2. 22-50 keV folded light curve of SAX J1818.6-1703 with a period of $30.0 \pm 0.2 \mathrm{~d}$ and a start time of IJD 1133.7365. Crosses correspond to $\sim 2 \mathrm{ks}$ flares, and boxes and $5 \sigma$ upper-limits correspond to deep mosaics (typical exposure of $\sim 100 \mathrm{ks}$ ).

During the $0.0-0.2$ phase range, the average $22-50 \mathrm{keV}$ flux in mosaics varied between $(2-8) \times 10^{-11} \mathrm{erg} \mathrm{cm}^{-2} \mathrm{~s}^{-1}$. During short flares, the source usually reached fluxes of $\sim(2-6) \times$ $10^{-10} \mathrm{erg} \mathrm{cm}^{-2} \mathrm{~s}^{-1}$ and, for very few flares, it even reached $\sim(1-2) \times 10^{-9} \mathrm{erg} \mathrm{cm}^{-2} \mathrm{~s}^{-1}$. During an XMM-Newton observation corresponding to phase $\sim 0.58$ (i.e. near apastron passage), the $0.5-10 \mathrm{keV} 3 \sigma$ upper-limit was $<10^{-13} \mathrm{erg} \mathrm{cm}^{-2} \mathrm{~s}^{-1}$. Therefore, the compact object must move on an eccentric orbit where it crosses the accretion zone only for $6 \mathrm{~d}$. Only during this phase did SAX J1818.6-1703 behave as an SGXB.

Let us consider the classical accretion model for SGXBs assuming a smooth wind. The semi-major axis $a$ of the system is determined with $a^{3} / P^{2}=G\left(M_{*}+M_{\mathrm{X}}\right) / 4 \pi^{2}$ with the period $P$, the stellar mass $M_{*}$, and the mass of the compact object fixed to $M_{\mathrm{X}}=1.4 M_{\odot}$. The stellar wind velocity of a supergiant can be approximated as $v_{\text {wind }}(r)=v_{\infty}\left(1-R_{*} / r\right)^{\beta}$ where $v_{\infty}$ is the terminal velocity, $R_{*}$ the stellar radius, and $\beta=0.8-1.2$. The stellar mass-loss rate $\dot{M}_{\text {wind }}=\dot{M}_{\text {wind }}\left(L_{*}, M_{*}, v_{\infty} / v_{\text {esc }}, T_{\text {eff }}\right)$ is determined using Eq. (12) of Vink et al. (2000) where $v_{\text {esc }}$ is the stellar escape velocity, and $T_{\text {eff }}$ the stellar effective temperature. Accretion can occur within a radius $R_{\text {accr }}$ determined as $R_{\text {accr }}=$ $2 G M_{\mathrm{X}} /\left(v_{\text {wind }}^{2}+v_{\mathrm{X}}^{2}\right)$ where $v_{\mathrm{X}}$ is the compact object velocity. The accretion rate $\dot{M}_{\text {accr }}$ is determined as $\dot{M}_{\text {accr }}=\left(R_{\text {accr }}^{2} / 4 a^{2}\right) \dot{M}_{\text {wind }}$. The luminosity $L_{\mathrm{X}}$ can be approximated as $L_{\mathrm{X}}=\epsilon \dot{M}_{\mathrm{accr}} c^{2}$ with $\epsilon \sim 0.2$ the accretion efficiency. Considering typical stellar fundamental parameters of O9I-B1I stars (Martins et al. 2005; Crowther et al. 2006), the expected accretion rates vary only by a factor of 2-3 between spectral types. However, they strongly vary with the distance between the star and the compact object with a factor of $\sim 10$ between $r=2-3 R_{*}$. The expected fluxes when $r=3 R_{*}$ are consistent with the low flux detected for SAX J1818.6-1703 by the different missions. Therefore, we assumed that SAX J1818.6-1703 starts to accrete when entering the zone within $r \leq 3 R_{*}$. Still, the size of the accretion zone may vary up to $1 R_{*}$ as the stellar mass-loss rate can change by a factor of 2-3 in supergiants with identical spectral type (Crowther et al. 2006). 

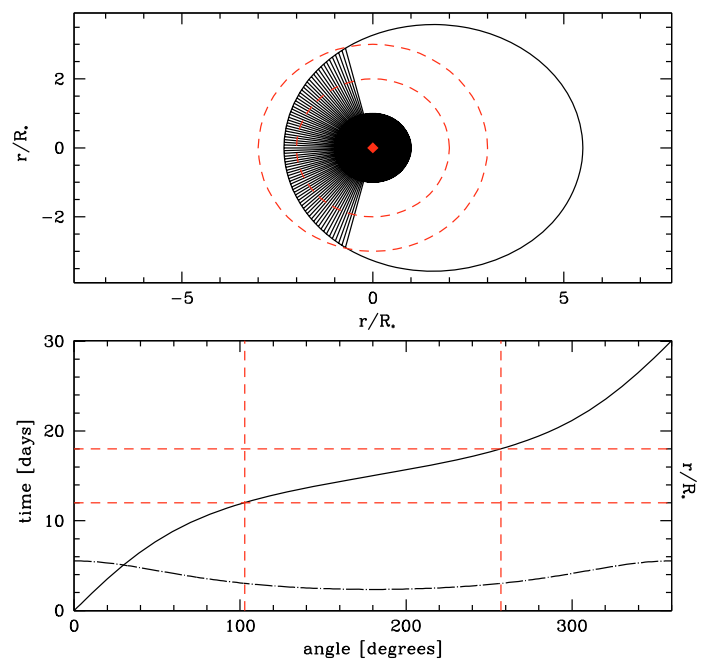

Fig. 3. Top: SGXB with elliptical orbit (solid line), B0.5I star (solid circle), and $e=0.41$. Circular dashed lines defined for $r=2$ and $3 R_{*}$. The accretion zone $\left(r \leq 3 R_{*}\right)$ is highlighted. Bottom: the solid line is the time of the compact object (left axis) along the orbit $(t=0 \mathrm{~d}$ is aphelion); dot-dashed line is the distance star-compact object (right axis) along the orbit; and the light dashed lines determine the accretion zone where $r \leq 3 R_{*}$ and $t \in t_{\text {accr }}$.

Under this condition and considering an elliptical orbit ${ }^{2}$, the period and the elapsed time of accretion $t_{\text {accr }}$ derived above implies constraints on the binary system eccentricity $e$. For O9I, O9.5I and B0Ia stars, the maximum elapsed accretion times allowed by the stellar parameters are $2.8,3.0$, and $4.6 \mathrm{~d}$ with $e=0.70, e=0.69$, and $e=0.58$, respectively, that are inferior to the $t_{\text {accr }}$ derived above. For a B0.5Ia star, the conditions are fulfilled with $e=0.41$ that implies a perihelion and aphelion of 2.3 and $5.5 R_{*}$ (see Fig. 3) or $e=0.58$ with $a \in 1.6-6.2 R_{*}$, respectively. For a B1Ia star, the conditions are fulfilled with $e=0.25$ (i.e. $a \in 2.7-4.5 R_{*}$ ) or $e=0.73$ (i.e. $a \in 1.0-6.2 R_{*}$ ). Only B0.5Ia and B1Ia stars seem to fill the accretion condition observed in SAX J1818.6-1703. Discarding the short and bright flare detections, the X-ray flux varies between $\sim(2-8) \times$ $10^{-11} \mathrm{erg} \mathrm{cm}^{-2} \mathrm{~s}^{-1}$ along the whole $t_{\text {accr }}$. Therefore, the compact object distance $r$ should not vary by a high factor when crossing the accretion zone, i.e. low eccentricities are favoured. Therefore, the cases of a B0.5-1Ia companion star with a low eccentricity ( $e=0.41$ or 0.25 , respectively) are the best solutions to reconcile the wind model and observations. This determination is accurate to 0.5 spectral type since an accretion zone of $4 R_{*}$ would also give a solution for a B0I star with $e=0.16$.

Short and bright flares were also observed that were 10-100 times higher than the average level during the accretion phase, and $>10^{3-4}$ outside this phase. in't Zand (2005) and Walter \& Zurita Heras (2007) showed that such huge flares in transient SGXBs resulted from the accretion of massive clumps formed within the stellar wind. Indeed, it was shown that instabilities of the radiation line-driven outflows of hot stars generated structure within the stellar wind, affecting parameters such as the wind velocity, the outflow density, or the mass-loss rate (Runacres \& Owocki 2002, and references therein). Recent studies of supergiant X-ray/UV line emission have shown that optically-thick macro-clumps must form within the wind of hot supergiant stars (Oskinova et al. 2007, and references therein). Determining average parameters of luminosity, frequency, and

2 The Kepler's 2nd law allows to determine $t=t(e, a, \theta(r))$. duration of flares in SFXTs from INTEGRAL observations, Walter \& Zurita Heras (2007) derived clump parameters that agreed with this macro-clump scenario developed by Oskinova et al. (2007), and predicted that accretion in SGXBs should follow a two-regime behaviour depending on whether the distance between the compact object and the supergiant companion was higher or lower than $\sim 2-3 R_{*}$. Negueruela et al. (2008) also expected the same behaviour and showed that the number of clumps that a compact object could statistically accrete within one orbit around a O8-B0I star varies between 1-8 with an orbital radius $a=3-6 R_{*}$. These values agree with the observation of SAX J1818.6-1703 flares, and can explain the observation of flares slightly outside the accretion phase, such as the flare observed by BeppoSAX.

Acknowledgements. JAZH thanks Farid Rahoui, J. Rodriguez, D. M. Smith and R. Walter for useful discussions on SAX J1818.6-1703. The authors also acknowledge the use of NASA's Astrophysics Data System.

Note added in proof. After the presentation of our results at the 7th INTEGRAL workshop (Zurita Heras et al., in press) and after the submission of our paper, Bird et al. (2008) confirmed our discovery.

\section{References}

Bird, A. J., Bazzano, A., Hill, A. B., et al. 2008, ArXiv e-prints Bodaghee, A., Walter, R., Zurita Heras, J. A., et al. 2006, A\&A, 447, 1027 Bodaghee, A., Courvoisier, T. J.-L., Rodriguez, J., et al. 2007, A\&A, 467, 585 Bozzo, E., Campana, S., Stella, L., et al. 2008, The Astronomer's Telegram, 1493, 1

Chaty, S., Rahoui, F., Foellmi, C., et al. 2008, A\&A, 484, 783

Crowther, P. A., Lennon, D. J., \& Walborn, N. R. 2006, A\&A, 446, 279

Dean, A. J., Bazzano, A., Hill, A. B., et al. 2005, A\&A, 443, 485

Filliatre, P., \& Chaty, S. 2004, ApJ, 616, 469

Götz, D., Falanga, M., Senziani, F., et al. 2007, ApJ, 655, L101

Grebenev, S. A., \& Sunyaev, R. A. 2005, Astron. Lett., 31, 672

Grebenev, S. A., \& Sunyaev, R. A. 2008, The Astronomer's Telegram, 1482, 1 Horne, J. H., \& Baliunas, S. L. 1986, ApJ, 302, 757

in't Zand, J. J. M. 2005, A\&A, 441, L1

in 't Zand, J., Heise, J., Smith, M., et al. 1998, IAU Circ., 6840, 2

in't Zand, J., Jonker, P., Mendez, M., \& Markwardt, C. 2006, The Astronomer's Telegram, 915, 1

Kuulkers, E., Shaw, S. E., Paizis, A., et al. 2007, A\&A, 466, 595

Lebrun, F., Leray, J. P., Lavocat, P., et al. 2003, A\&A, 411, L141

Leyder, J.-C., Walter, R., Lazos, M., Masetti, N., \& Produit, N. 2007, A\&A, 465, L35

Lutovinov, A., Revnivtsev, M., Gilfanov, M., et al. 2005, A\&A, 444, 821

Markwardt, C. B., Swank, J. H., Marshall, F. E., \& in't Zand, J. J. M. 2000, in Rossi2000: Astrophysics with the Rossi X-ray Timing Explorer, ed. T. E. Strohmayer

Martins, F., Schaerer, D., \& Hillier, D. J. 2005, A\&A, 436, 1049

Negueruela, I., \& Smith, D. M. 2006, The Astronomer's Telegram, 831, 1

Negueruela, I., \& Schurch, M. P. E. 2007, A\&A, 461, 631

Negueruela, I., Smith, D. M., Reig, P., Chaty, S., \& Torrejón, J. M. 2006, in The X-ray Universe 2005, ed. A. Wilson, ESA SP-604, 165

Negueruela, I., Torrejón, J. M., Reig, P., Ribó, M., \& Smith, D. M. 2008, in American Institute of Phys. Conf. Ser., 1010, 252

Oskinova, L. M., Hamann, W.-R., \& Feldmeier, A. 2007, A\&A, 476, 1331

Pellizza, L. J., Chaty, S., \& Negueruela, I. 2006, A\&A, 455, 653

Press, W. H., \& Rybicki, G. B. 1989, ApJ, 338, 277

Revnivtsev, M. G., Sunyaev, R. A., Varshalovich, D. A., et al. 2004, Astron. Lett., 30, 382

Rodriguez, J., Bodaghee, A., Kaaret, P., et al. 2006, MNRAS, 366, 274

Runacres, M. C., \& Owocki, S. P. 2002, A\&A, 381, 1015

Sguera, V., Barlow, E. J., Bird, A. J., et al. 2005, A\&A, 444, 221

Smith, D. M., Heindl, W. A., Markwardt, C. B., et al. 2006, ApJ, 638, 974

Ubertini, P., Lebrun, F., Di Cocco, G., et al. 2003, A\&A, 411, L131

Vink, J. S., de Koter, A., \& Lamers, H. J. G. L. M. 2000, A\&A, 362, 295

Walter, R., \& Zurita Heras, J. 2007, A\&A, 476, 335

Walter, R., Zurita Heras, J., Bassani, L., et al. 2006, A\&A, 453, 133

Winkler, C., Courvoisier, T. J.-L., Di Cocco, G., et al. 2003, A\&A, 411, L1

Zurita Heras, J. A., de Cesare, G., Walter, R., et al. 2006, A\&A, 448, 261 